\title{
Size and shape of graphene layers in commercial carbon blacks established by Debye refinement
}

\author{
Yuri G Andreev ${ }^{\mathrm{a} *}$ and Peter G Bruce ${ }^{\mathrm{b}}$

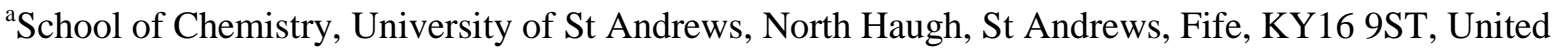 \\ Kingdom \\ ${ }^{\mathrm{b}}$ Department of Materials, University of Oxford, Park Road, Oxford, OX1 3PH, United Kingdom
}

Correspondence email: ya@st-andrews.ac.uk

Synopsis Geometry of graphene layers in two commercial carbon blacks has been established from powder diffraction data using refinement based on Debye equation. The ordered regions within the layers are curved and essentially non-circular.

Abstract The size and the shape of graphene layers in commercial conductive carbon blacks, Super $\mathrm{P} \circledast$ and Super $\mathrm{S} 囚$, have been determined from powder $\mathrm{X}$-ray diffraction data. Using a refinement procedure based on the fundamental diffraction equation of Debye, it is shown that the ordered regions within the layers of both materials are of elliptical shape, curved in a cylindrical fashion along the longer axis of the ellipse. The regions are greater in Super $\mathrm{P} 囚$, ellipse axes: 5.4 and $2.2 \mathrm{~nm}$, than in Super $\mathrm{S} \circledast$, 4.6 and $2.1 \mathrm{~nm}$, and less curved, curvature radii: 12.7 and $11.7 \mathrm{~nm}$ respectively. There is no crystallographic registry between layers that are equidistantly stacked into concentric groups of 6 or 7 , on average.

Keywords: Graphene, powder diffraction, Debye equation.

\section{Introduction}

Graphitic materials with different morphologies and various degrees of inter- and intra-layer ordering are widely used in industry and scientific research. Carbon blacks, obtained through incomplete combustion or decomposition of hydrocarbons and characterized by a large surface area coupled with turbostratic disorder of graphene layers,(Palomares et al., 2010) find their main applications as pigments and reinforcing agents. However, in recent years Super $S \circledR$ and Super $P ®$ carbon blacks are extensively utilized as electronically conductive light-weight additives for battery electrodes. Since the conductivity of graphitic materials arises from the electron delocalization in the $\pi$-electron 
honeycomb network of carbon atoms, the size and shape of the layers must play an important part in determining intrinsic conduction properties.

Establishing the geometry of one-atom-thick layers grouped together, often in disorderly fashion, to form particles of a powder, is a challenging task. Transmission electron microscopy (TEM) readily provides only profile images of the layers and cannot ensure proper averaging over the bulk of a powdered material. Powder diffraction methods offer good averaging but the analysis of the data is complicated by significantly asymmetric peak shapes originating from intra-layer diffraction of independently scattering aromatic sheets and Bragg intensities that cannot be described using one unit cell. A number of approaches have been developed over the last 60 years which provide means of extracting structural information from such patterns. Non-overlapping intra-layer reflections from honeycomb arrangements of atoms are readily approximated by peak shape functions derived using either the fundamental scattering equation of Debye(Debye, 1915; Diamond, 1957; Warren \& Bodenstein, 1965) or the structure-amplitude approach,(Wilson, 1949; Warren \& Bodenstein, 1966) providing a means of determining the size of flat circular aromatic layers and the in-plane lattice parameter.(Andreev \& Lundstrom, 1994) Using Fourier analysis of peak shapes of carbon black powders, which utilizes approximation of crystallites by ellipsoids, it is possible to extract the lateral crystallite diameter of the layers together with the median and variance of the size distribution, as well as the density of dislocations.(Ungar et al., 2002) Whole-pattern fitting procedures, which use theoretical expressions for both intra- and inter-layer diffraction peaks derived by employing a number of asymptotes and approximations, have been shown to provide average layer and (layer) stack sizes along with parameters characterizing polydispersity of both sizes, parameters of disorder, homogeneity of the stacks, strains, etc., up to 14 parameters in total.(Shi et al., 1993; Ruland \& Smarsly, 2002) Atomic pair distribution function (PDF) analysis of nanoporous carbons reveals curvature of the graphene layers and suggests a tentative microscopic model of the local structure.(Petkov et al., 1999)

The above methodological approaches provide a wealth of information about the structure of disordered graphites but do not produce a clear impression of the size and the shape of graphene layers in these materials. Here we report a refinement method which delivers a scaled model of the average ordered graphene region in a turbostratically distorted graphite using the shape of an in-plane reflection in a powder diffraction pattern. The method is illustrated using two commercial carbon black materials.

\section{Experimental}

Powders of Super $\mathrm{S} \circledast$ and Super $\mathrm{P} \circledast$ carbon blacks were purchased from TIMCAL and used as received. 
X-ray powder diffraction patterns were collected on a PANalytical Empyrean diffractomer equipped with a copper anode tube, primary beam monochromator and the $\mathrm{X}^{\prime}$ Celerator ${ }^{\mathrm{TM}}$ detector. The powders were placed on a zero-background silicon substrate and the patterns collected for $8 \mathrm{hrs}$ in Bragg-Brentano geometry, continuous scan mode with recorded step size of $0.0170^{\circ}$ within $5-100^{\circ} 2 \theta$ range.

Transmission electron microscopic images were acquired on a JEOL JEM-2011 electron microscope fitted with a LaB6 filament operating at an accelerating voltage of $200 \mathrm{kV}$. The TEM images were recorded using a Gatan 794 CCD camera.

\section{Results}

\subsection{Preliminary analysis of powder diffraction patterns}

Powder diffraction patterns of Super $S \circledR$ and Super $P ®$ are shown in Fig. 1 together with a calculated pattern of ideal graphite. On visual inspection of the diffraction data it becomes apparent that the carbon blacks have the structure of a turbostratically disordered graphite.

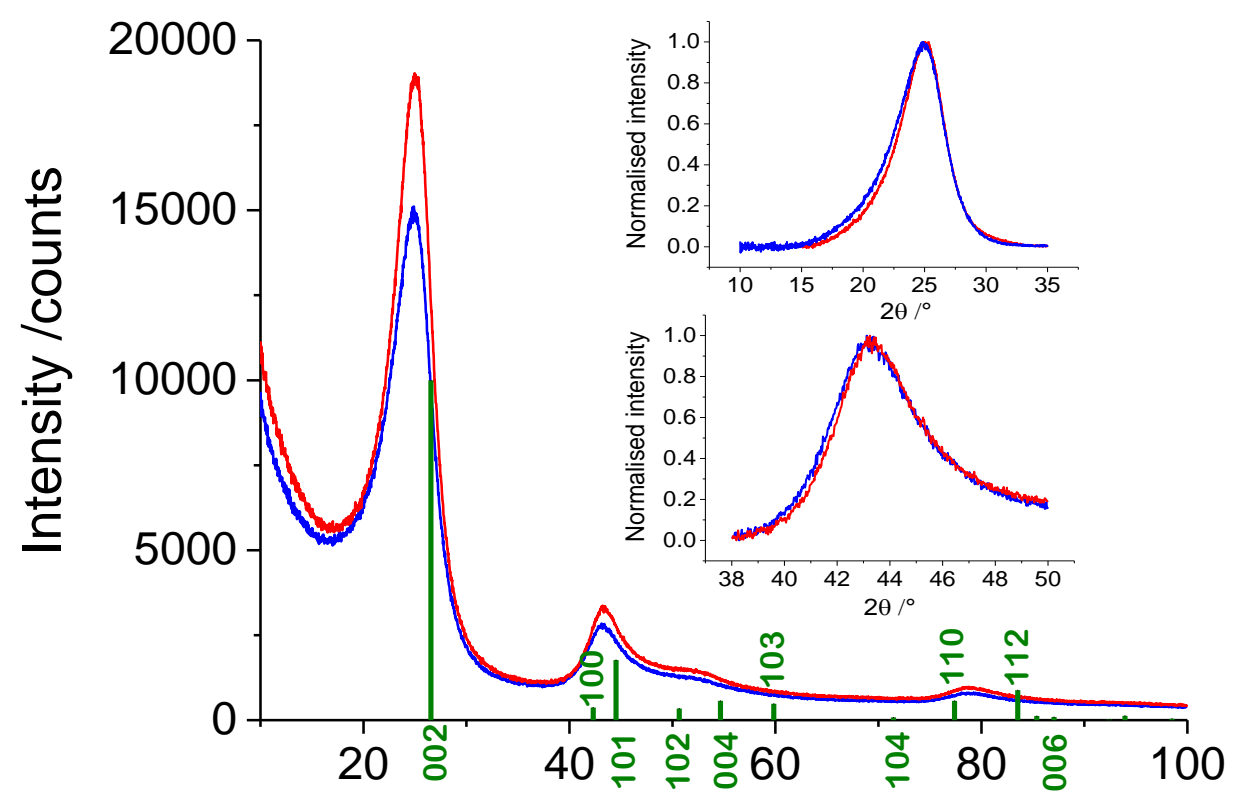

Figure 1

$2 \theta / 0$

Powder diffraction patterns of Super $\mathrm{P} \circledast$ (red) and Super $\mathrm{S} \circledast$ (blue). Green vertical lines and associated numbers represent positions, intensities and Miller indices $(h k l)$ of graphite reflections. Insets show background-subtracted and normalized on the maximum intensity count parts of the diffraction profiles representing most prominent inter- (top) and intra-layer (bottom) reflections.

The first peak, which is affiliated with the 002 reflection in the ideal structure, is broad and shifted to a lower $2 \theta$ angle, suggesting presence of only few equidistant graphene layers which lack extended planarity, leading to greater inter-layer distance. Traces of the higher orders, 004 and 006, of the same 
reflection can also be found within the measured angular range (see Fig. 1). The nonappearance of h01 and hkl reflections in the patterns of Super $S \circledR$ and Super $P ®$ indicates absence of the graphitic registry between the layers. Further evidence of the violation of 3D crystallographic registry is provided by the shape of the peak at $\sim 43.3^{\circ}$, akin to the in-layer 100 reflection in graphite, which is similar in appearance to the asymmetric shape of reflections from a single aromatic layer.(Diamond, 1957; Warren \& Bodenstein, 1965; Warren \& Bodenstein, 1966; Wilson, 1949) In addition, the width and the position of the maximum count of this peak, which now has to be assigned as a 10 reflection, suggest that the size of the ordered regions within the layers is smaller than $10 \mathrm{~nm}$.(Andreev \& Lundstrom, 1995) Thus, the diffraction patterns of the two carbon blacks contain features that are associated with two types of ordering that can no longer be described using a single unit cell. Such decoupling allows selective extraction of information about the inter- and intra-layer order from the representative parts of the patterns.

The powder diffraction profiles of Super $\mathrm{S} \circledast$ and Super $\mathrm{P} \circledast$ are very similar. However, a closer inspection, see the insets in Fig.1, reveals that the inter-layer peak is broader in the pattern of Super $\mathrm{S} 囚$, indicating shorter average stack of equidistant layers. The distance between layers, calculated from the peak position, appears to be the same in both powders, $0.355 \mathrm{~nm}$, and greater than in graphite, $0.3356 \mathrm{~nm}$. The height of the stack, estimated from the width of the peak using the Scherrer equation, is $10 \%$ greater in Super $\mathrm{P} \circledast, 2.2 \mathrm{~nm}$ compared to $2.0 \mathrm{~nm}$ in Super $\mathrm{S} \circledast$, which translates into stacks comprising on average 7 and 6.5 equidistant graphene layers respectively.

From the inset in Fig. 1 showing the markedly asymmetric intra-layer 10 reflection in the carbon blacks it is clear that the latter is broader in Super $\mathrm{S} \circledast$. The complexity of diffraction peak shapes from 2D-ordered structures precludes easy determination of structural parameters' values using the position of maximum count or width of the reflection. In fact, unlike for 3D-ordered structures, the angular position of the maximum is no longer a function of only the in-plane lattice parameters but also depends on the in-plane crystallite size, while the latter has a pronounced effect on the overall peak shape.(Andreev \& Lundstrom, 1995) Evaluation of the crystallite size has been attempted on the assumption that the graphene layers are flat and circular, with $a$ lattice parameter of graphite, 0.2464 $\mathrm{nm}$, by calculating diffraction profiles from individual layers of different diameter using the equation of Debye.(Debye, 1915) As evident from Fig.2, such model does not adequately describe the observed profile. In order to match the experimental data a refinement procedure must be employed, with both size and shape variable parameters.

\subsection{Refinement procedure}

The refinement is aimed at finding the best fit to the experimental data, $y^{o b s}(2 \theta)$, of the diffraction profile, calculated using the Debye equation $y^{c a l}(2 \theta)$, from a single graphene sheet, by varying 


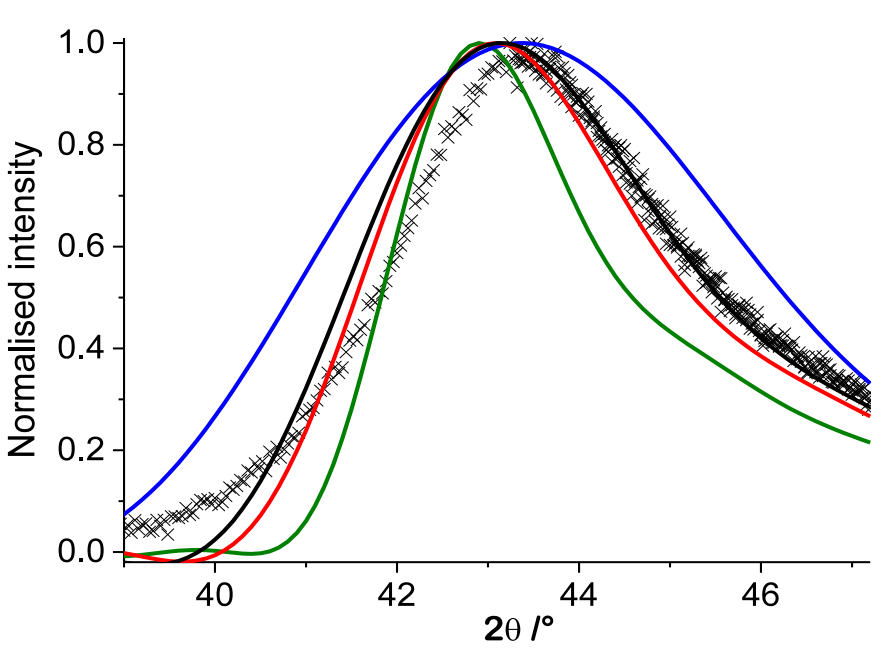

\section{Figure 2}

Diffraction profiles of the 10 reflection. Crosses - experimental data (Super P®); solid lines calculated using Debye equation from a single flat circular graphene layer with diameter 5 (green), 3.5 (red), 3 (black) and 2 (blue) nm.

parameters defining its shape and size, as well as the interatomic distances within the aromatic layer. The quality of fit is assessed in the least-squares sense using the $\chi^{2}$ criterion:

$$
\chi^{2}=\frac{1}{N-N_{p}} \sum_{i=1}^{N} \frac{\left(y_{i}^{o b s}\left(2 \theta_{i}\right)-y_{i}^{c a l}\left(2 \theta_{i}\right)\right)^{2}}{y_{i}^{o b s}\left(2 \theta_{i}\right)}
$$

where $N$ and $N_{p}$ are the number of profile points and the number of variable parameters, respectively. The values of $y_{i}^{c a l}\left(2 \theta_{i}\right)$ for each trial graphene sheet are calculated using the Debye equation:

$$
y_{i}^{c a l}\left(2 \theta_{i}\right)=N_{a} f^{2}\left(2 \theta_{i}\right)+2 f^{2}\left(2 \theta_{i}\right) \sum_{k=1}^{N_{a}} \sum_{m=1}^{N_{a}}\left[\sin \left(4 \pi r_{k m} \sin \theta_{i} / \lambda\right) /\left(4 \pi r_{k m} \sin \theta_{i} / \lambda\right)\right]
$$

$N_{a}$ - number of atoms in the sheet; $f\left(2 \theta_{i}\right)$-X-ray form-factor for carbon; $r_{k m}$ - distance between the $k^{\text {th }}$ and the $m^{\text {th }}$ atoms within the sheet.

Coordinates of individual atoms, using which an array of $r_{k m}$ can be readily obtained, within each trial graphene sheet, using the current values of the parameters, are calculated as follows.

- Coordinates of carbon atoms within a 2D unit cell of ideal aromatic layer are determined using the in-plane lattice parameter, $a$.

- The coordinates from the previous step are translated 40 times along the $a$ and $b$ axes of the unit cell.

- The array of coordinates is reduced to those of the atoms located within an ellipse, with the current lengths $A$ and $B$ of the axes, drawn around the geometrical centre of the sheet. The $A$ axis of the ellipse is positioned at an angle, $\gamma$, with respect to the [10] direction within the 2D lattice, Appendix A. 
- The coordinates from the previous step are modified to map cylindrically or spherically bent sheet using the curvature radius, $R$.

Minimization of the $\chi^{2}$, by varying the values of $a, A, B, \gamma$ and $R$, is carried out by a simulated annealing algorithm, which is similar to those reported earlier.(Andreev et al., 1997; Andreev et al., 2014) The refinement procedure is coded in C++ for a multicore LINUX workstation, with the computation of the double sum in the Debye equation performed by the processors in parallel, Appendix B.

\subsection{Outcome of refinement}

The refinement was performed using the region of the diffraction pattern containing the 10 reflection using cylindrical or spherical bending of a flat elliptical graphene sheet. The region with the 11 reflection was not used due to its very lower intensity, larger overlap with the neighbouring 006 peak (compared to 10 and 004). Incorporation of the 11 peak into the refinement would have also required variation of the amplitudes of the mean-squares displacement of the atoms within the layer (to account for the fade of intensity with $2 \theta$ ) which more than likely would lead to unreliable values of the amplitudes, due to the low statistical significance of the 11 , giving rise to correlations with other variables and invalidating the latter. The results are given only for the cylindrical curvature, since it provides a better fit to the experimental diffraction data for both carbons, Fig.3.
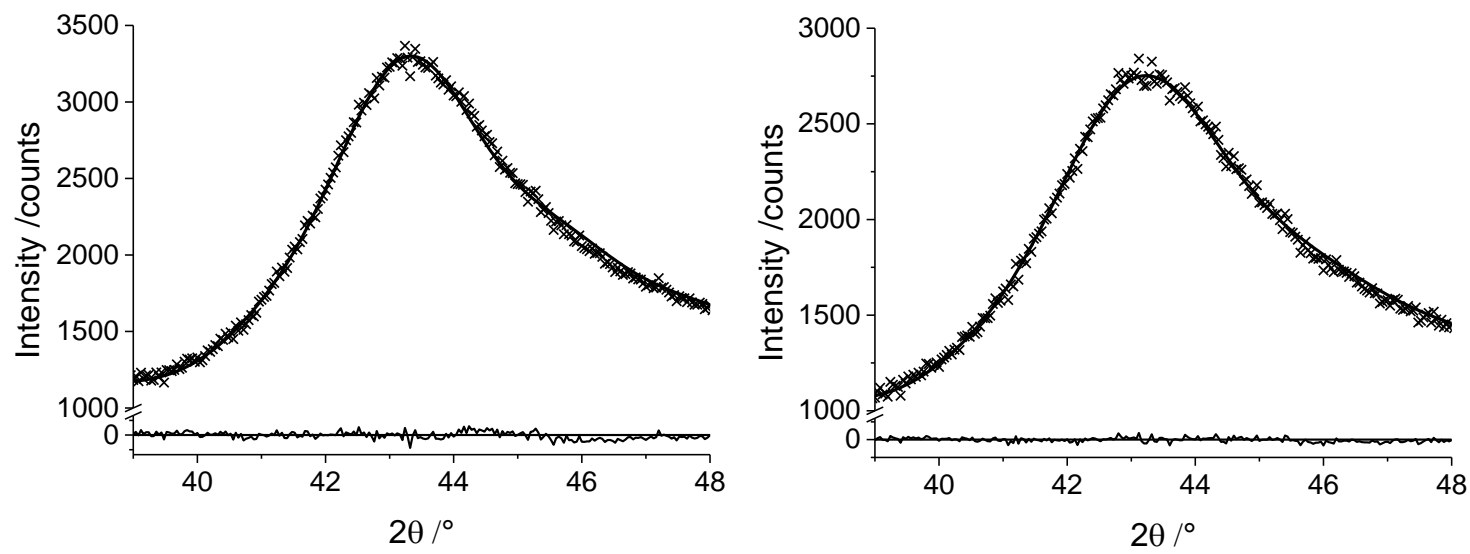

\section{Figure 3}

Observed (line), calculated (crosses) and difference (line) powder diffraction profiles of the 10 reflection from Super $P \circledast$ (left) and Super $S \circledR$ (right) at the end of the refinement

Starting and refined values of variable parameters are listed in Table I. The same set of starting parameters was used in both refinements. The minimizations reduce $\chi^{2}$ from the initial value of 8.7 to 1.1 (Super $\mathrm{P} 囚$ ) and from 2.3 to 0.6 (Super $\mathrm{S} \circledast$ ), while fully exploring the parameter space (see the range of $\chi^{2}$ values in Fig. 4). 

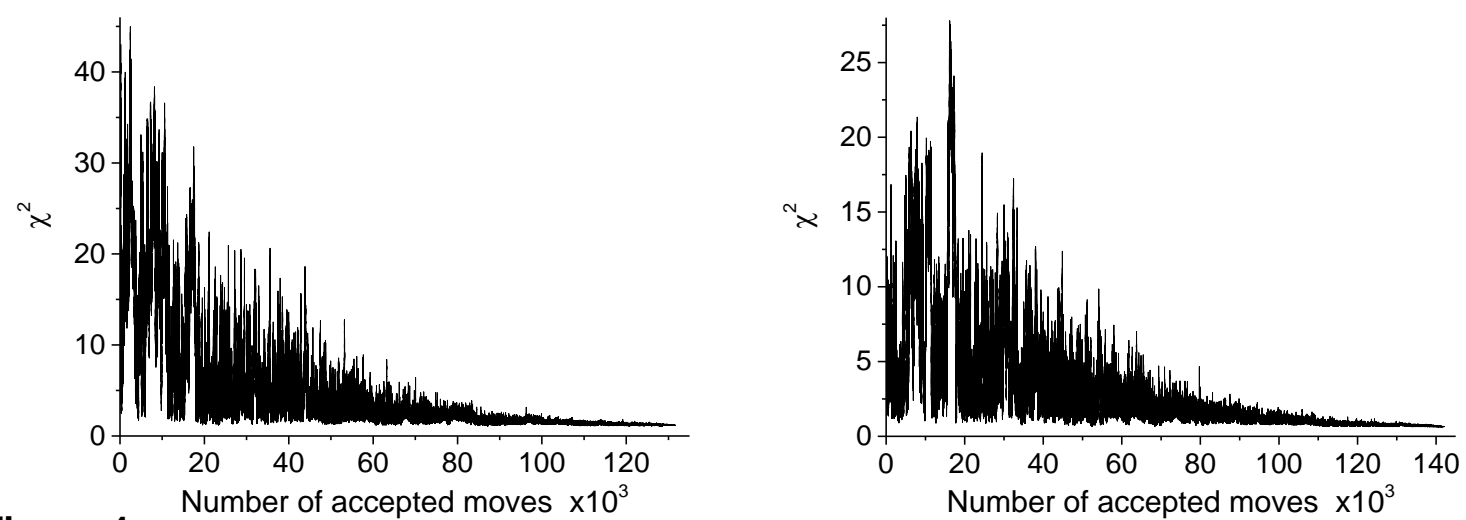

Figure 4

$\chi^{2}$ values as function of the number of accepted moves (trial set of variable parameters) for Super $\mathrm{P} \circledR$ (left) and Super $\mathrm{S} \circledast$ (right)

Table 1 Initial and refined values of variable parameters plus investigated ranges. See text for details. Errors in the refined values obtained through multiple refinements and amount to 1 in the last decimal place.

\begin{tabular}{llllll}
\hline & $\boldsymbol{a}(\mathbf{n m})$ & $\boldsymbol{A}(\mathbf{n m})$ & $\boldsymbol{B}(\mathbf{n m})$ & $\boldsymbol{R}(\mathbf{n m})$ & $\gamma\left(^{\circ}\right)$ \\
\hline Initial & 0.2464 & 3 & 3 & 30 & 0 \\
Range & $0.245-0.248$ & $2-8$ & $2-8$ & $0-50$ & $0-60$ \\
Super P® & 0.2461 & 2.21 & 5.40 & 12.7 & 1.022 \\
Super $\mathrm{S} 囚$ & 0.2466 & 2.12 & 4.55 & 11.7 & 1.020 \\
\hline
\end{tabular}

As can be seen from Table I, the in-plane lattice parameter within the ordered regions of both samples does not significantly deviate from the value in graphite, and the orientation of the sheet with respect to the crystallographic axis is the same for Super $\mathrm{P} \circledast$ and Super $\mathrm{S} \circledast$. The ordered regions in both carbons are essentially non-circular and slightly non-planar, Fig. 5. The greater extent of sheet Super $P \circledast$, together with a higher value of the curvature radius, explains the observed difference in the breadth of the 10 reflection between the two carbon blacks (see the lower inset in Fig. 1). The ordered region in Super $\mathrm{P} \circledast$ contains 362 atoms, compared to 290 in Super $\mathrm{S} \circledast$.
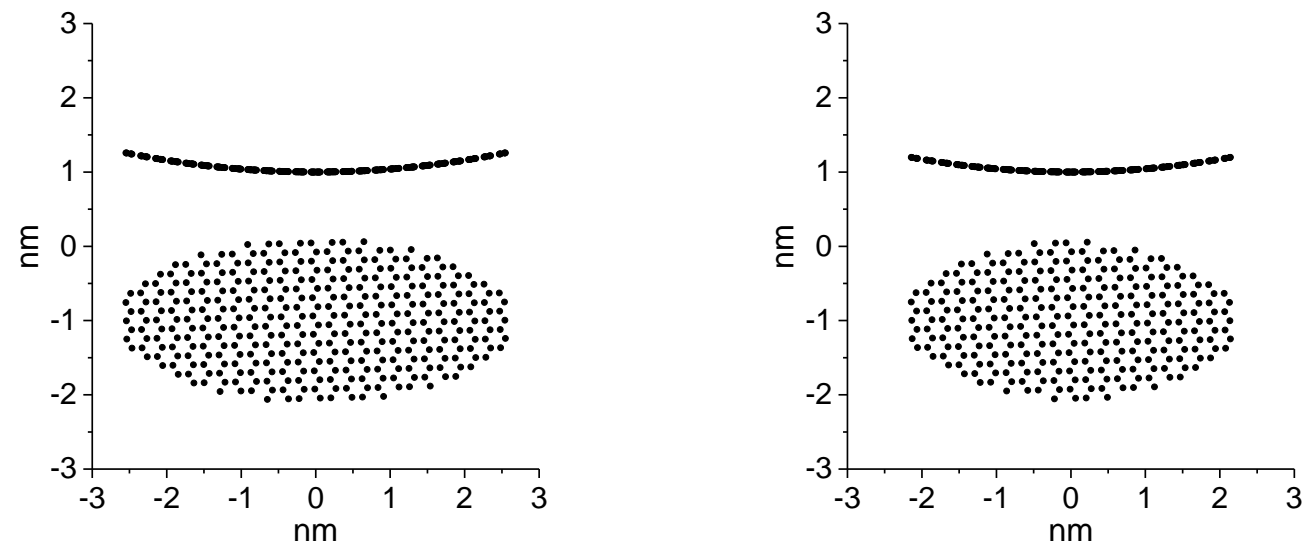


\section{Figure 5}

Plan (bottom) and profile (top) views of the in-plane ordered regions in Super P® (left) and Super $\mathrm{S} \circledast$ (right)

\section{Discussion}

According to the TEM images, Fig. 6, both powders consist of spherical particles $30-60 \mathrm{~nm}$

The results of the powder diffraction analysis indicate that the investigated carbon black powders contain regions with, on average, 7 equidistant graphene layers that are not in crystallographic registry with each other. The shape of an average ordered region within each layer can be approximated by an ellipse with distinctly different axes lengths. In addition, the layers are slightly curved along the longer axis. The curvature is readily mapped by a cylindrical surface. The ordered regions are smaller and their curvature is slightly higher in Super $S \circledR$.

The established curvature of the layers does alter ideal carbon-carbon distances in an aromatic arrangement. The carbon sheets are strained, with the distances gradually changing when moving from the centre of the layer to its periphery. However, the maximum deviation from the nearest neighbours' separation in graphite, $0.1423 \mathrm{~nm}$, found at the edges of the ellipse in Super $\mathrm{P} \circledast, 0.1370$ nm, is well within the reported limit of $96 \%$ for graphene sheets.(Warner et al., 2013) According to the TEM images, Fig. 6, both powders consist of spherical particles $30-60 \mathrm{~nm}$ in diameter. At higher resolution, the curvature of the graphene layers is clearly seen in Fig. 6, with parts of the particle resembling segments of an onion. Some of the layers observed in the TEM images appear to be greater than the length of the longest axis of the ellipse obtained in the course of the Debye refinement. However, only sections of the profile of those layers cannot be represented by a single arc, whose curvature and extent agree with the powder diffraction results (see yellow lines in Fig. 6).

Combination of powder diffraction and TEM results leads to the conclusion that particles of Super $\mathrm{P} \circledast$ and Super $\mathrm{S} \circledast$ consist of turbostratic graphene layers containing cylindrically curved elliptical coherently scattered regions. Some of the ordered regions from different layers are arranged into concentric equidistant stacks, with layer separation greater than in graphite and no crystallographic registry, except in the radial direction.

The exact nature of the Debye equation allows extraction of structural information using every point in the observed powder diffraction pattern, while direct comparison with raw experimental data assures confidence in the results obtained through structure refinement. In the case of commercial 
carbon blacks reported here such approach reveals structural variations manifested by subtle difference in the diffraction profiles.
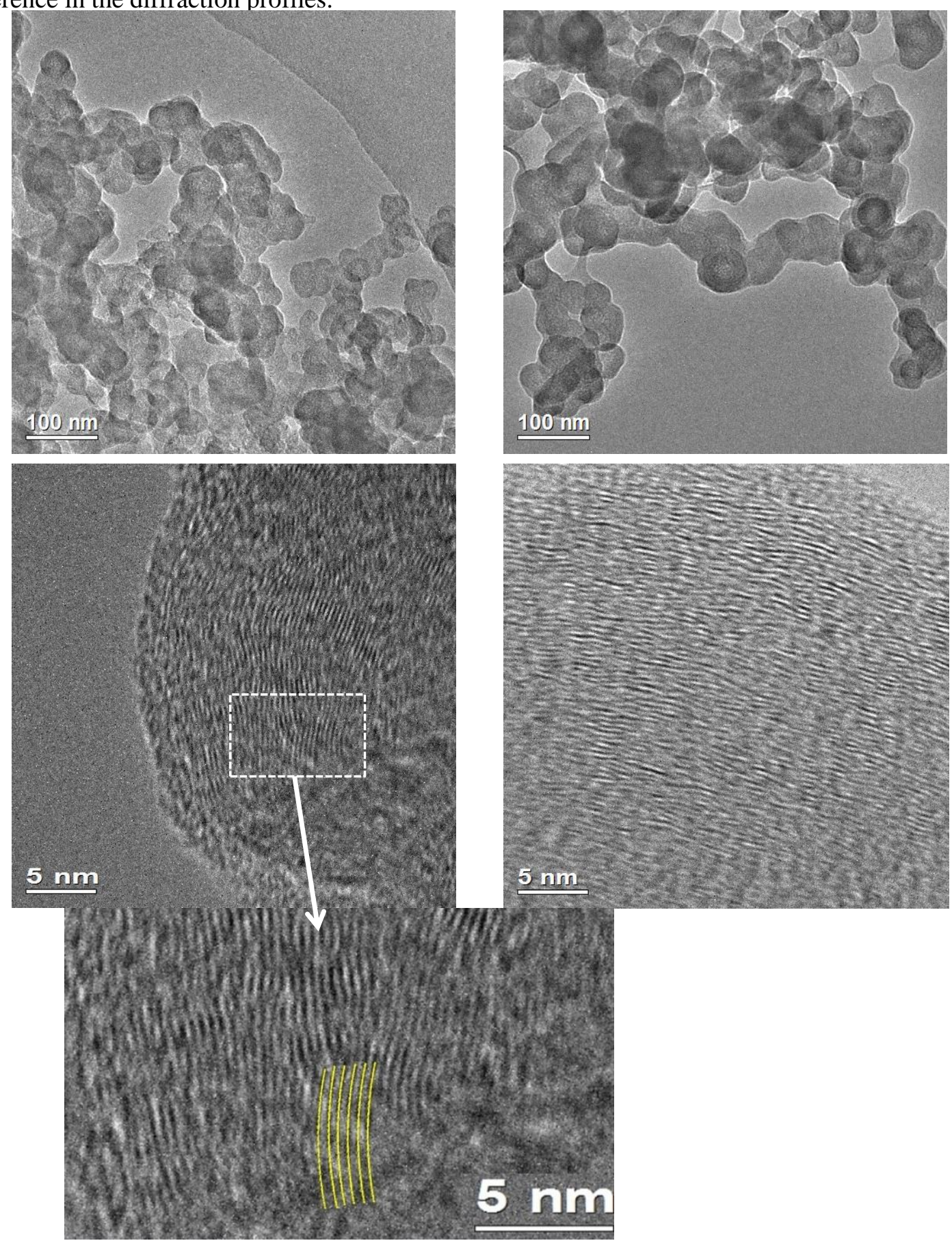

\section{Figure 6}

TEM images of Super $P \circledR$ (left) and Super $\mathrm{S} \circledast$ (right). Yellow lines in the cut-out (bottom image) represent layer profiles, obtained from Debye refinement and shown on the same scale, which exactly match the observed fringes.

\section{Conclusion}


The exact nature of the Debye equation allows extraction of structural information using every point in the observed powder diffraction pattern, while direct comparison with raw experimental data assures confidence in the results obtained through structure refinement. It has been demonstrated that information about the structure of the layers in layered compounds lacking 3D order can be obtained by using Debye refinement utilising only the relevant region of the diffraction pattern. In the case of commercial carbon blacks reported here such approach reveals structural variations manifested by subtle difference in the diffraction profiles. The developed refinement procedure is applicable to a wide range of materials with limited extent of translational symmetry. The refinement can be extended to larger-scale models by employing computers with greater number of processors or by utilising graphics processing units, suggested by the group of P. Scardi.(Gelisio et al., 2010). However, it has to be noted that applications to structures with essentially greater, than in the given examples, extent of the ordered regions will require integral convolution of the pattern calculated using the equation of Debye with the instrumental broadening shapes, in order to compare with the experimental data.

Acknowledgements Authors are grateful to Dr Heather Greer for acquiring the TEM images. PGB acknowledges financial of EPSRC, including the SUPERGEN project.

\section{References}

Andreev, Y. G., Lightfoot, P. \& Bruce, P. G. (1997). J. Appl. Crystallogr. 30, 294-305.

Andreev, Y. G. \& Lundstrom, T. (1994). J. Appl. Crystallogr. 27, 767-771.

Andreev, Y. G. \& Lundstrom, T. (1995). J. Appl. Crystallogr. 28, 534-539.

Andreev, Y. G., Panchmatia, P. M., Liu, Z., Parker, S. C., Islam, M. S. \& Bruce, P. G. (2014). J. Am. Chem. Soc. 136, 6306-6312.

Debye, P. (1915). Annalen Der Physik 46, 809-823.

Diamond, R. (1957). Acta Crystallographica 10, 359-364.

Gelisio, L., Azanza Ricardo, C. L., Leoni, M. \& Scardi, P. (2010). J. Appl. Crystallogr. 43, 647-653.

Palomares, V., Goni, A., Gil de Muro, I., de Meatza, I., Bengoechea, M., Cantero, I. \& Rojo, T. (2010). J. Power Sources 195, 7661-7668.

Petkov, V., DiFrancesco, R. G., Billinge, S. J. L., Acharya, M. \& Foley, H. C. (1999). Philosophical Magazine B-Physics of Condensed Matter Statistical Mechanics Electronic Optical and Magnetic Properties 79, 1519-1530.

Ruland, W. \& Smarsly, B. (2002). J. Appl. Crystallogr. 35, 624-633.

Shi, H., Reimers, J. N. \& Dahn, J. R. (1993). J. Appl. Crystallogr. 26, 827-836.

Ungar, T., Gubicza, J., Ribarik, G., Pantea, C. \& Zerda, T. W. (2002). Carbon 40, 929-937.

Warner, J. H., Lee, G. D., He, K., Robertson, A. W., Yoon, E. \& Kirkland, A. I. (2013). ACS Nano 7, 9860-9866.

Warren, B. E. \& Bodenstein, P. (1965). Acta Crystallographica 18, 282-286.

Warren, B. E. \& Bodenstein, P. (1966). Acta Crystallographica 20, 602-605.

Wilson, A. J. C. (1949). Acta Crystallographica 2, 245-251. 


\section{Appendix A. Geometry, position and curvature of a graphene layer}

In order to achieve flexible positioning of the cut-out-ellipse axes, the atoms in the uncut graphene sheet are first rotated clockwise by a variable angle $\gamma$.

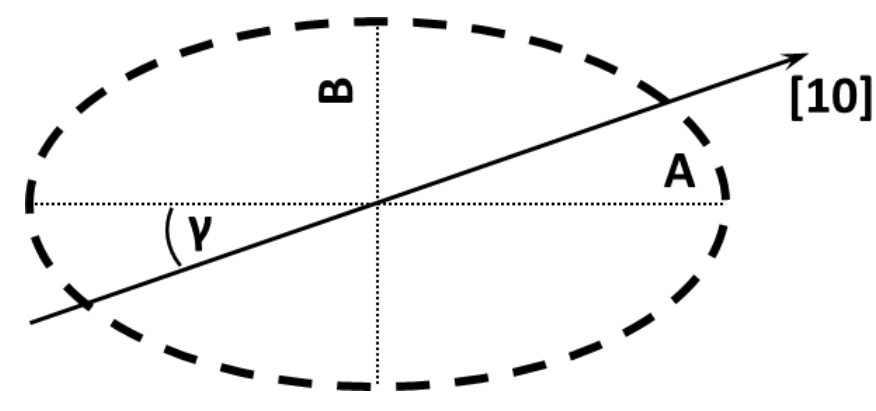

The position of the A axis coincides with the [10] direction prior to rotation:

$x_{i}=x_{0} \cos \gamma-y_{0} \sin \gamma ; \quad y_{i}=x_{0} \sin \gamma+y_{0} \cos \gamma$.

Only those atoms whose coordinates fulfill the condition:

$x_{i}^{2} / A^{2}+y_{i}^{2} / B^{2} \leq 1$

are considered in a trial model at each combination of $\gamma, \mathrm{A}$ and B.

Elliptical bending of the resulting graphene sheet is achieved by using the following transformation of the coordinates:

$y_{i}^{\prime}=R \sin \beta ; z_{i}^{\prime}=R(1-\cos \beta) ; \beta=y_{i} / R$

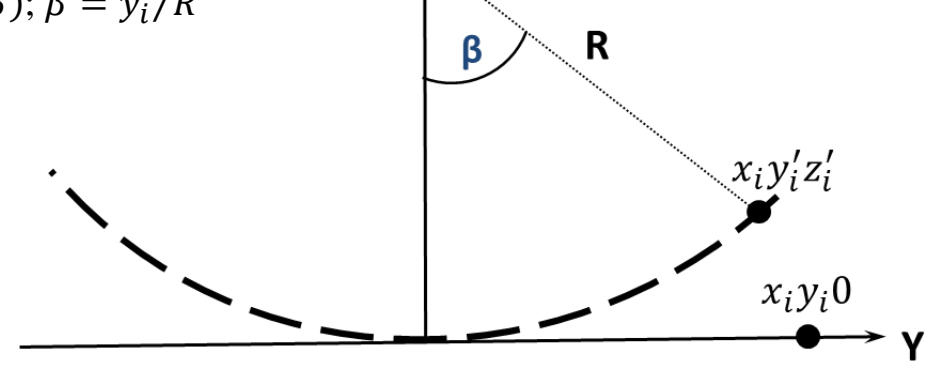

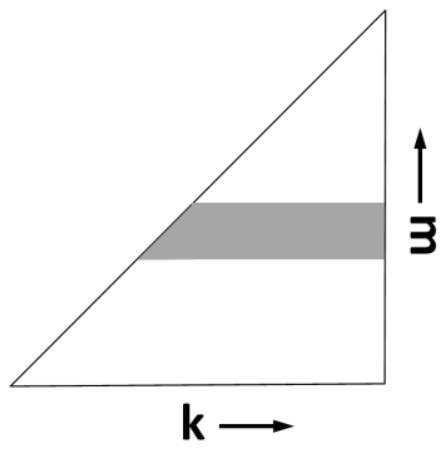

\section{Appendix B. Distribution of computational load between} processors

The total computational load of Eg. 2 can be represented by the area of a triangle, since each interatomic distance has to be taken into account 3 only once. Thus, the goal of evenly distributing the calculation of the double sum between $\mathrm{N}_{\mathrm{p}}$ processors working in parallel is achieved by dividing the triangle into $\mathrm{N}_{\mathrm{p}}$ trapezoids with the same area. Each of the 
processors calculates the part of the double sum within the boundaries of the indices determined by such geometrical split. 